\title{
Cortical somatosensory processing measured by magnetoencephalography predicts neurodevelopment in extremely low-gestational-age infants
}

\author{
Petri Rahkonen ${ }^{1}$, Päivi Nevalainen², Leena Lauronen³, Elina Pihko ${ }^{4}$, Aulikki Lano 5 , Sampsa Vanhatalo ${ }^{3}$, Anu-Katriina Pesonen ${ }^{6}$, \\ Kati Heinonen ${ }^{6}$, Katri Räikkönen ${ }^{6}$, Leena Valanne ${ }^{7}$, Taina Autti ${ }^{7}$, Sture Andersson ${ }^{1}$ and Marjo Metsäranta ${ }^{1}$
}

BACKGROUND: Higher cortical function during sensory processing can be examined by recording specific somatosensoryevoked magnetic fields (SEFs) with magnetoencephalography (MEG). We evaluated whether, in extremely low-gestationalage (ELGA) infants, abnormalities in MEG-recorded SEFs at term age are associated with adverse neurodevelopment at 2 y of corrected age.

METHODS: SEFs to tactile stimulation of the index finger were recorded at term age in 30 ELGA infants $(26.5 \pm 1.2 \mathrm{wk}$, birth weight: $884 \mathrm{~g} \pm 181 \mathrm{~g}$ ). Neurodevelopment was evaluated at 2 y of corrected age. Controls were 11 healthy term infants.

RESULTS: In nine of the ELGA infants (30.0\%), SEFs were categorized as abnormal on the basis of lack of response from secondary somatosensory cortex (SII). At $2 y$, these infants had a significantly worse mean developmental quotient and locomotor subscale on the Griffiths Mental Development Scales than the ELGA infants with normal responses. Mild white matter abnormalities in magnetic resonance imaging at term age were detected in $21 \%$ of infants, but these abnormalities were not associated with adverse neurodevelopment.

CONCLUSION: Abnormal SII responses at term predict adverse neuromotor development at 2 y of corrected age. This adverse development may not be foreseen with conventional neuroimaging methods, suggesting a role for evaluating SII responses in the developmental risk assessment of ELGA infants.

B ecause of the increasing number of extremely low-gestational-age (ELGA) survivors, more attention is being paid to early detection of brain injury and abnormal brain development to enable early interventions for the prevention of longterm morbidity (1). At present, however, ELGA infants often develop with motor, sensory, cognitive, and behavioral impairments (2-4).

Grade III and IV intraventricular hemorrhages (IVHs) and cystic periventricular leukomalacia are associated with adverse neurodevelopmental outcome. However, neurodevelopmental problems are frequently encountered in infants with normal cranial ultrasonography (US) (5). Compared with US, conventional magnetic resonance imaging (MRI) provides more information on white matter (WM) (6), and moderate to severe WM injury predicts adverse neurodevelopmental outcome (7-10). However, neurodevelopmental problems also occur in preterm infants with normal MRI $(10,11)$.

Because the structural imaging methods partly fail in detecting the milder abnormalities that may compromise later neurocognitive development of ELGA infants, additional methods are needed to predict neurodevelopmental outcome and allow the early commencement of appropriate rehabilitation. Previous neurophysiological studies assessing the functioning of the somatosensory system in preterm infants have focused on the first cortically generated component in the somatosensory-evoked potentials (SEPs) with the aim of assessing the integrity of the pathways from the periphery to the cortex (12-15). These studies have been motivated by the idea that somatosensory pathways run through the vulnerable periventricular zone and hence the SEPs could reflect the otherwise elusive functional integrity in that structure.

It has recently become apparent, however, that a prevalent type of preterm brain injury is a diffuse WM injury associated with a variety of secondary disturbances in the structural and functional brain organization (16). This has led to a call for methods that would directly assess integrity of cortical functions beyond the primary sensory areas, thereby providing more elaborate information on the functional intactness of developing cortical networks.

Magnetoencephalography (MEG) is a noninvasive method for detecting weak extracranial magnetic fields produced by currents generated in the cerebral cortex. Activity from both the primary somatosensory cortex (SI) and the secondary somatosensory cortex (SII) can be detected and modeled in both newborn term infants and ELGA infants at term

\footnotetext{
'Department of Pediatrics, Children's Hospital, Helsinki University Central Hospital, University of Helsinki, Helsinki, Finland; ${ }^{2}$ BioMag Laboratory, Helsinki Medical Imaging Center, Helsinki University Central Hospital, University of Helsinki, Helsinki, Finland; ${ }^{3}$ Department of Clinical Neurophysiology, Children's Hospital, Helsinki University Central Hospital, University of Helsinki, Helsinki, Finland; ${ }^{4}$ Brain Research Unit, O.V. Lounasmaa Laboratory, Aalto University School of Science, Espoo, Finland; ${ }^{5}$ Department of Child Neurology, Children's Hospital, Helsinki University Central Hospital, University of Helsinki, Helsinki, Finland; ${ }^{6}$ Faculty of Behavioral Sciences, University of Helsinki, Helsinki, Finland; ${ }^{7}$ Helsinki Medical Imaging Center, University of Helsinki, Helsinki, Finland. Correspondence: Petri Rahkonen (petri.rahkonen@hus.fi)
} 


\section{Articles $\mid$ Rahkonen et al.}

equivalent age (TEA) (17-19). Because MEG can detect somatosensory processing beyond primary areas, we wanted to test whether the abnormalities in cortical processing of somatosensory information in ELGA infants, reflected as
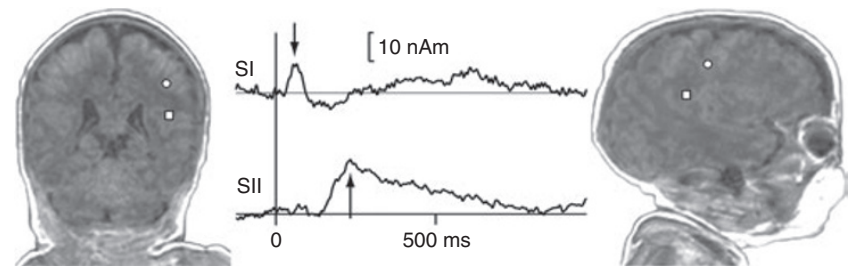

Figure 1. Strength and location of activity in the right primary $(\mathrm{SI})$ and secondary (SII) somatosensory cortices to stimulation of the left index finger. The open spheres indicate the SI source and the open squares indicate the SII source in one extremely low-gestational-age infant. The upper line shows how the strength of activity in SI changes in time with its peak at $\sim 60 \mathrm{~ms}$ (arrow), and the lower line shows the strength of the activity in SII with its peak at $240 \mathrm{~ms}$. The vertical line denotes the time of stimulation. abnormal somatosensory-evoked magnetic fields (SEFs) at TEA, are associated with adverse neurodevelopmental outcome at $2 \mathrm{y}$ of corrected age.

\section{RESULTS}

\section{MEG and Neurodevelopmental Outcome}

The SEF response from the contralateral SI, peaking at $\sim 60 \mathrm{~ms}$ after tactile stimulation, was detectable in all ELGA infants and controls (Figure 1). The M60 peak latencies and source strengths were normal (within $2.5 \mathrm{SD}$ from the mean reported in full-term newborns) (20) in all but one preterm infant, who showed a slightly delayed M60. The response from the contralateral SII (Figure 2), peaking at $\sim 200 \mathrm{~ms}$ after stimulus, was absent in nine ELGA infants in the right hemisphere and eight in the left hemisphere. To ipsilateral stimulation, the SII response was visible in the right hemisphere in three of the nine infants and in the left hemisphere in three of the eight infants with response missing to contralateral
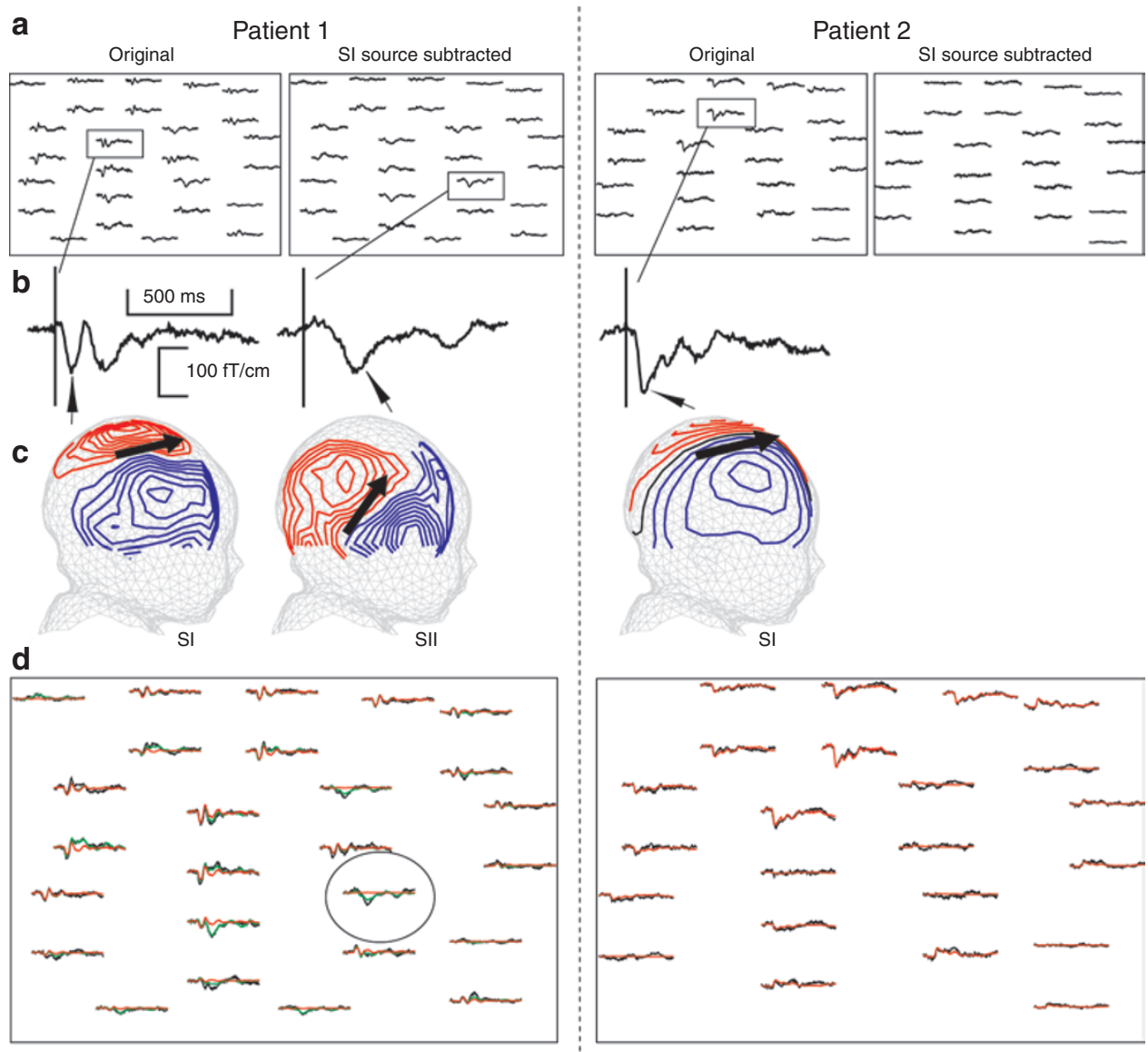

Figure 2. Examples of preterm infants with normal secondary somatosensory cortex (SII) response (Patient 1) and missing SII response (Patient 2). (a) For both infants, the upper left hand panel shows the responses of a subset of magnetoencephalography gradients over the measured hemisphere. $(\mathbf{b}, \mathbf{c})$ In both infants, the first deflection can be modeled with an equivalent current dipole (ECD) displayed in c, where the magnetic contour maps are reflected on a spherical surface. The red lines indicate magnetic field exiting the head, and blue lines indicate magnetic field entering the head. The arrow represents the ECD. Note that the ECD for the first deflection has a horizontal orientation and is located centrally. The ECD orientation and location agree with source location at the primary somatosensory cortex $(\mathrm{SI})$. When this SI source is subtracted from the data (a: on the upper right hand panel for both infants), another deflection can be seen in the data of Patient 1 (as in $\mathbf{a}, \mathbf{b})$, but not in Patient 2. (c) This later deflection can be modeled with an ECD with upright orientation and location more inferiorly and laterally (as compared with the SI ECD), agreeing with source location at the SII in the parietal operculum. (d) The modeled waveforms (red: one-dipole model including SI ECD, green: two-dipole model including SI and SII ECDs) are on top of the measured waveforms (black). Note that the one-dipole model (red) fits well with the data of Patient 2 but does not explain the later deflection for Patient 1 , whereas the two-dipole model (green) also explains the later deflection for Patient 1 (red vs. green line on circled channel). 


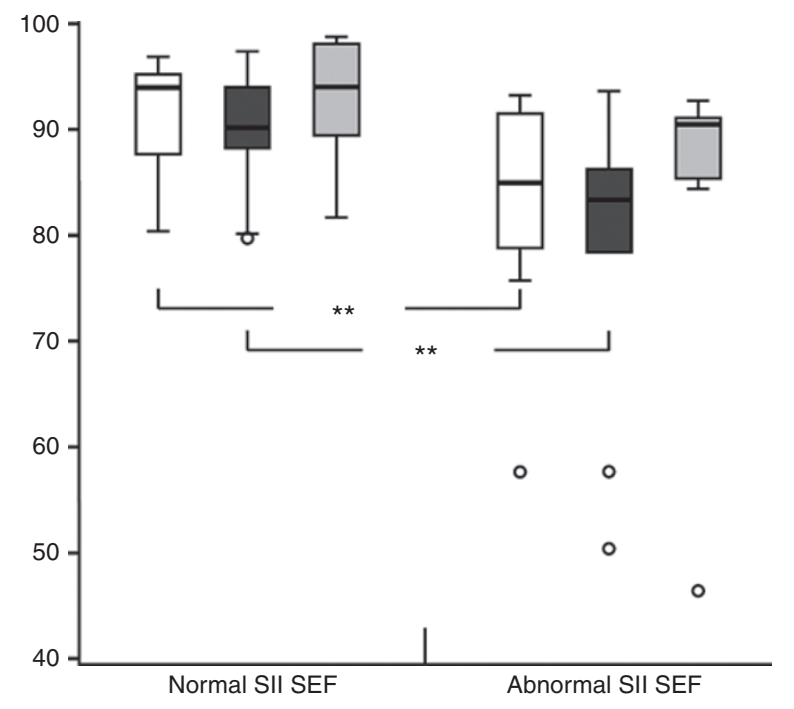

Figure 3. Griffiths Mental Development Scales (29) quotients in extremely low-gestational-age infants with normal (left) and abnormal secondary somatosensory cortex (SII) response in magnetoencephalography (MEG). White boxes: developmental quotient; dark gray boxes: locotomor subscale; lighter gray boxes: eye-hand coordination. The dark lines in the middle of the boxes depict the median, and open circles show outliers. ${ }^{* *} P<0.01$. SEF, somatosensory-evoked magnetic field.

stimulation. When, in either hemisphere (right or left), both contra- and ipsilaterally evoked SII responses were absent, the SEF was considered to be abnormal. Consequently, the SII SEF was abnormal in nine of 30 ELGA infants. Of the control infants, one showed an absence of SII responses in the right hemisphere (left not recorded), and this infant also showed minor WM abnormality in MRI (focal regions of high T1- or T2-weighted signal). His developmental quotient (DQ) on the Griffiths Mental Development Scales (GMDS) and cognition and language development on the Bayley Scales of Infant and Toddler Development, Third Edition (BSID-III), were lower than those of controls on average. In infants in whom SII responses were present, the peak latencies and source strengths were within normal limits (mean $\pm 2.5 \mathrm{SD})(20)$.

The ELGA infants with abnormal SII SEF responses had lower total DQ and locomotor subscale quotient (SQ) in GMDS $(P<0.01)$ than the ELGA infants with normal SII responses (total DQ: $82.8 \pm 11.2$ vs. $91.8 \pm 4.6$; locomotor subscale: $78.6 \pm 14.8$ vs. $90.0 \pm 4.8$, respectively; Figure 3 ). The difference in eye-hand coordination approached significance $(P=0.07)$. There was no difference in cognition and language skills in ELGA infants with normal or abnormal SII response (BSID-III). Clinical characteristics and previous medical history of ELGA infants with normal and abnormal SII response in MEG did not differ significantly (Table 1). The neurological examination was normal significantly more often in the ELGA infants with normal $(13 / 21,62 \%)$ than in those with abnormal $(2 / 9,22 \%)$ SII response $(P<0.05)$. The one infant with delayed M60 peak latency had a normal 2 y outcome examination. The results of GMDS and BSID-III at $2 \mathrm{y}$ of corrected age are shown in Table 2.
Table 1. Characteristics of ELGA infants with normal and abnormal SII response in MEG

\begin{tabular}{|c|c|c|c|}
\hline & $\begin{array}{l}\text { Normal SII } \\
\text { response } \\
(n=21)\end{array}$ & $\begin{array}{l}\text { Abnormal SII } \\
\text { response } \\
(n=9)\end{array}$ & $P$ \\
\hline $\begin{array}{l}\text { Gestational age } \\
\text { at birth (wk) }\end{array}$ & $26.5(1.3)$ & $26.5(0.8)$ & 0.98 \\
\hline Birth weight (g) & 841 (184.9) & $895(159.0)$ & 0.46 \\
\hline Male & $8[38.1]$ & $7[77.8]$ & 0.11 \\
\hline Cesarean section & $11[52.4]$ & $5[55.6]$ & 0.87 \\
\hline Chorioamnionitis & 7 [33.3] & $5[55.6]$ & 0.42 \\
\hline $\begin{array}{l}\text { Prenatal } \\
\text { betamethasone } \\
\text { (at least one dose) }\end{array}$ & $21[100]$ & $9[100]$ & 1.00 \\
\hline $\begin{array}{l}\text { Prenatal } \\
\text { betamethasone } \\
\text { (two doses) }\end{array}$ & $16[76.2]$ & $8[88.9]$ & 0.63 \\
\hline $\begin{array}{l}\text { Small for } \\
\text { gestational age }\end{array}$ & $8[38.1]$ & $2[22.2]$ & 0.67 \\
\hline RDS & $15[71.4]$ & $4[44.4]$ & 0.23 \\
\hline BPD at hours $36+0$ & $8[38.1]$ & $4[44.4]$ & 0.75 \\
\hline Septicemia & $6[28.6]$ & $3[33.3]$ & 0.79 \\
\hline IVH grade III-IV & $2[9.5]$ & $1[11.1]$ & 0.89 \\
\hline PVL in US & $0[0]$ & $0[0]$ & 1.00 \\
\hline NEC & $1[4.8]$ & $0[0]$ & 0.50 \\
\hline ROP & $4[19.1]$ & $2[22.2]$ & 0.84 \\
\hline $\begin{array}{l}\text { Hemodynamically } \\
\text { significant PDA }\end{array}$ & $15[71.4]$ & $5[55.6]$ & 0.40 \\
\hline $\begin{array}{l}\text { Postnatal use of } \\
\text { dexamethasone }\end{array}$ & $1[4.8]$ & $1[11.1]$ & 0.52 \\
\hline
\end{tabular}

Data shown as mean (SD) or $n$ [\%].

BPD, bronchopulmonar dysplasia; ELGA, extremely low gestational age; IVH, intraventricular hemorrhage; $\mathrm{MEG}$, magnetoencephalography; $\mathrm{NEC}$, necrotizing enterocolitis; PDA, patent ductus arteriosus; PVL, periventricular leukomalacia; $R D S$, respiratory distress syndrome; ROP, retinopathy of prematurity; SII, secondary somatosensory cortex; US, ultrasonography.

\section{MRI, US, and Neurodevelopmental Outcome}

The neurodevelopmental outcome of infants with WM abnormalities in MRI or IVH grades III-IV in US is shown in Table 3. WM in MRI was defined as normal in 23 ELGA infants (79\%) and mildly abnormal in 6 infants (21\%). There were no moderate-to-severe WM abnormalities in the study population. Neurodevelopmental outcome at $2 \mathrm{y}$ of corrected age did not differ between ELGA infants with or without mild WM abnormalities. Among six ELGA infants with WM abnormalities, only one had abnormal SII response in MEG. His DQ 91.5 was the second lowest of these six (range: 87.3-96.9) and locomotor SQ 85.0 was the lowest (range: 85.0-97.4). He had complex minor neurological dysfunction (MND-2), whereas of the ELGA infants with WM abnormalities but normal SII response, three had normal neurological examination and two had simple minor neurological dysfunction (MND-1).

Gray matter in MRI was classified as normal in all ELGA and term infants according to Woodward et al. (5). However, more 


\section{Articles $\mid$ Rahkonen et al.}

Table 2. MEG and neurodevelopmental outcome at 24 mo of corrected age

\begin{tabular}{|c|c|c|c|c|}
\hline & \multicolumn{3}{|c|}{ ELGA infants } & \multirow[b]{2}{*}{$\begin{array}{l}\text { Term controls } \\
\quad(n=11)\end{array}$} \\
\hline & $\begin{array}{l}\text { Normal SII response } \\
\quad(n=21)\end{array}$ & $\begin{array}{l}\text { Abnormal SII response } \\
\qquad(n=9)\end{array}$ & $P$ & \\
\hline GMDS locomotor SQ & $90.0(4.8)$ & $78.7(14.8)$ & $<0.01$ & $90.0(2.6)$ \\
\hline GMDS personal and social SQ & $91.3(6.5)$ & $84.2(9.9)$ & 0.08 & $90.8(5.1)$ \\
\hline GMDS performance SQ & $93.8(4.2)$ & $88.3(9.9)$ & 0.09 & $94.2(2.3)$ \\
\hline BSID-III cognition ${ }^{a}$ & $10.8(1.7)$ & $9.4(3.9)$ & 0.25 & $13.3(3.2)$ \\
\hline BSID-III language receptive ${ }^{b}$ & $12.6(2.2)$ & $10.4(4.9)$ & 0.15 & $14.3(3.1)$ \\
\hline BSID-III language expressive ${ }^{c}$ & $11.3(2.7)$ & $8.5(5.2)$ & 0.11 & $10.4(4.5)$ \\
\hline \multicolumn{5}{|c|}{$\begin{array}{l}\text { aData for six ELGA infants and two healthy term infants missing. }{ }^{b} \text { Data for seven ELGA infants and two healthy term infants missing. 'Data for eight ELGA infants and two healthy term } \\
\text { infants missing. }\end{array}$} \\
\hline
\end{tabular}

Table 3. WM in MRI, IVH grades III-IV in US, and neurodevelopmental outcome at 24 mo of corrected age

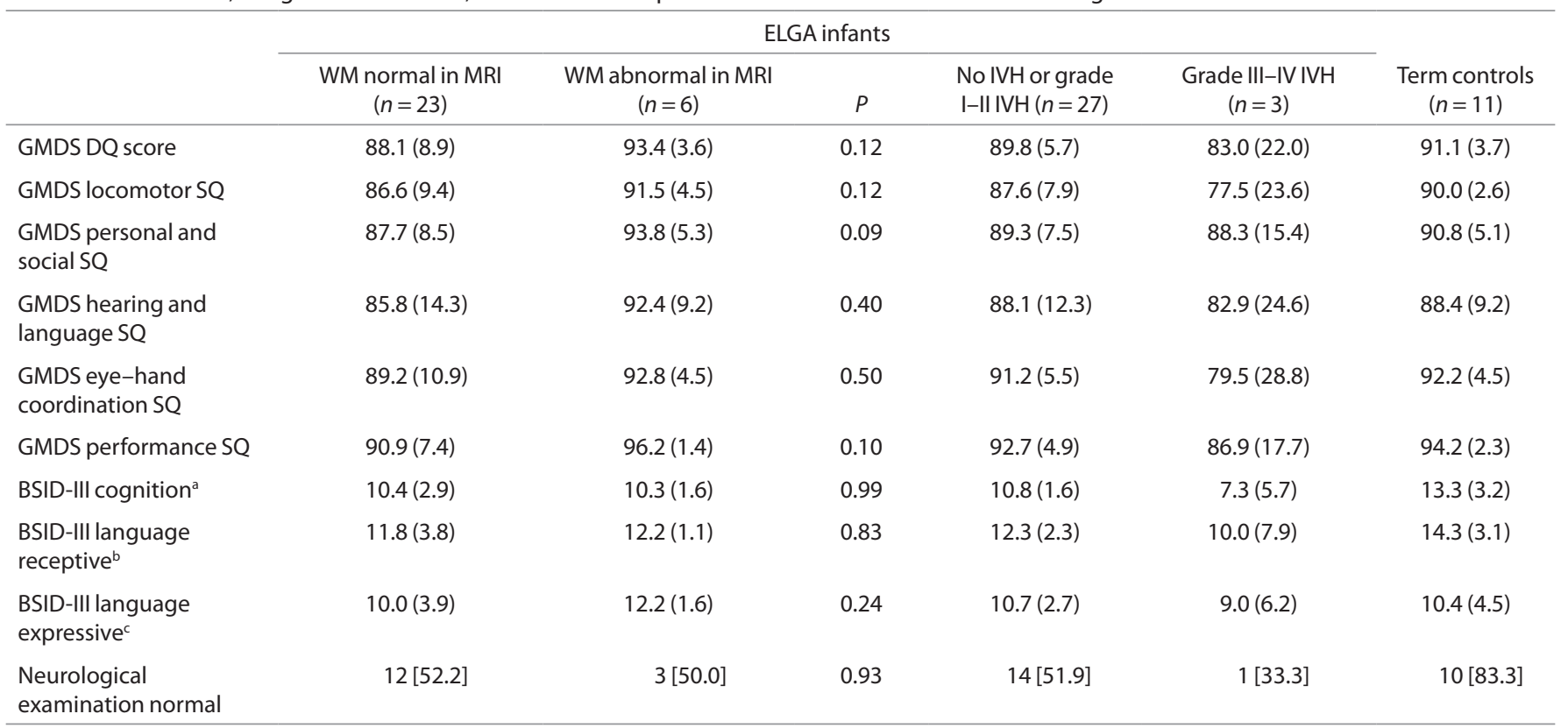

Data shown as mean (SD) or $n$ [\%].

BSID-III, Bayley Scales of Infant and Toddler Development, Third Edition; DQ, developmental quotient; ELGA, extremely low gestational age; GMDS, Griffiths Mental Development Scales; IVH, intraventricular hemorrhage; MRI, magnetic resonance imaging; SQ, subscale quotient; US, ultrasonography; WM, white matter.

aData for six ELGA infants and two healthy term infants missing. 'Data for seven ELGA infants and two healthy term infants missing. Data for eight ELGA infants and two healthy term infants missing.

subtle gray matter changes were found in 8 of 30 ELGA infants and 0 of 10 term infants.

Altogether, IVH in US was found in 8 of 30 ELGA infants; grade I-II IVH was present in five (16.7\%) and grade IIIIV IVH in three infants (10\%). Only two ELGA infants with IVH had abnormal SII response in MEG. Two ELGA infants with grade III-IV IVH had normal SII response in MEG, and their DQ was 96.9 and 94.5, locomotor SQ was
94.0 and 88.0, and neurological examination normal and MND-2, respectively. One infant with grade III-IV IVH and abnormal SII response in MEG had poor neurodevelopmental outcome with DQ 57.7, locomotor SQ 50.4, and neurological examination MND-2. The results of MEG, MRI, and US at TEA or neonatal period, and neurological examination at $2 \mathrm{y}$ of corrected age in ELGA infants are shown in Table 4. 
Table 4. MEG and neuroradiology at TEA and neurological outcome at $2 \mathrm{y}$ of corrected age in ELGA infants

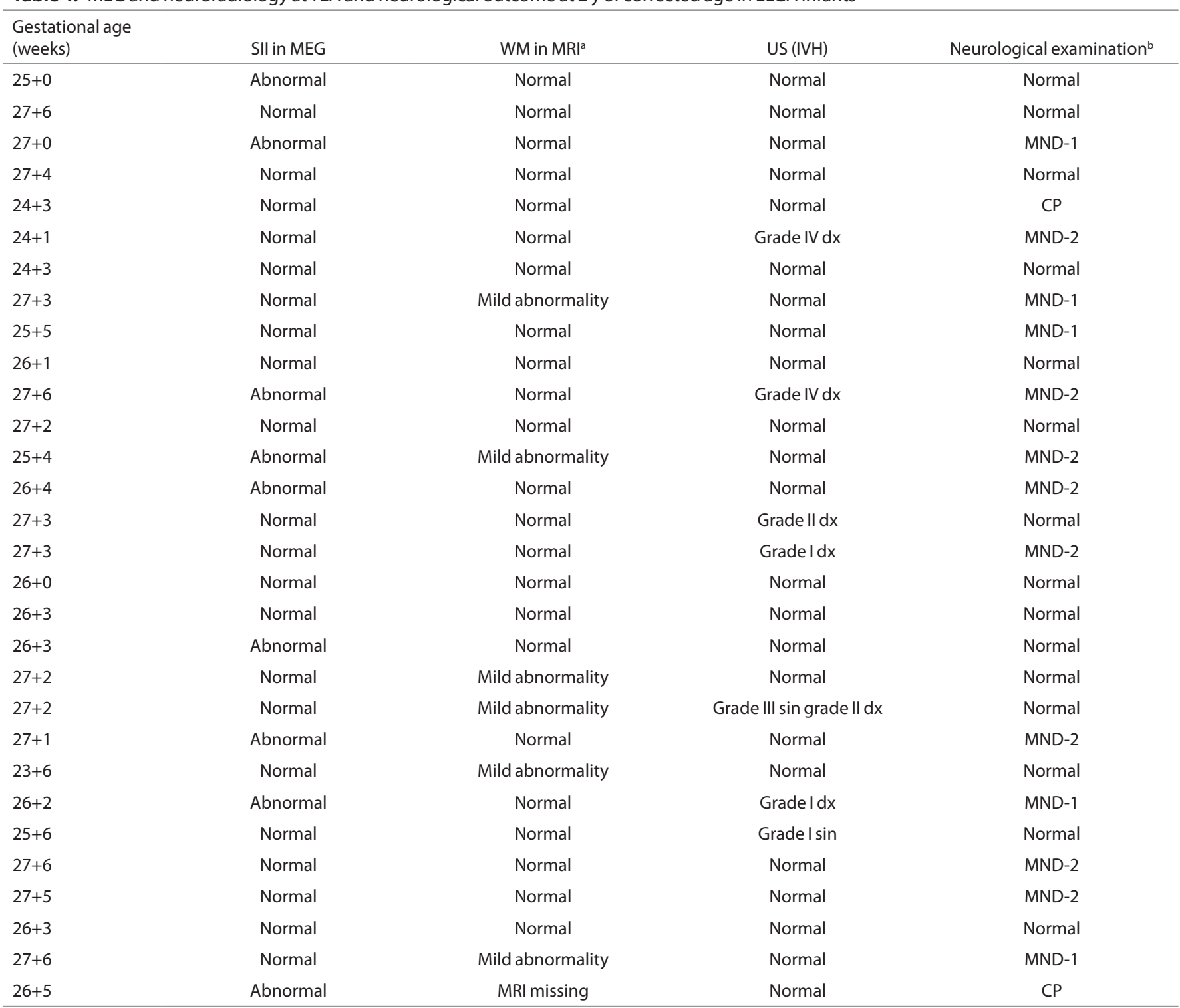

CP, cerebral palsy; dx, dexter (right); ELGA, extremely low gestational age; IVH, intraventricular hemorrhage; MEG, magnetoencephalography; MND-1, simple minor neurological dysfunction; MND-2, complex minor neurological dysfunction; MRI, magnetic resonance imaging; SIl, secondary somatosensory cortex; sin, sinister (left); TEA, term equivalent age; US, ultrasonography; WM, white matter.

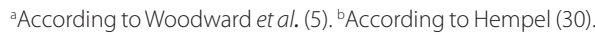

\section{DISCUSSION}

We show that late somatosensory responses from SII, which are associated with a higher level of somatosensory cortical processing, can be reliably studied with MEG in ELGA infants at term and that they may yield prognostic information overlooked by other neurophysiological and neuroimaging methods. We investigated whether abnormalities in SEFs are associated with problems in neurodevelopmental outcome of ELGA infants. Our results showed that the absence of SII responses at term age in ELGA infants is associated with poor mean DQ and locomotor subscale assessed by GMDS at $2 \mathrm{y}$ of corrected age. Furthermore, in this regard the SEF assessment surpassed MRI and cranial US in our study group.
Previously, somatosensory functions in infants have mainly been assessed using SEPs. Most previous preterm SEP studies have focused on the first cortical response as a predictor of sensorimotor outcome because it reflects the integrity of the somatosensory pathways from the periphery to the SI $(21,22)$. In the current study, as in a previous MEG study (18), SI response was seen in every infant, thus having no predictive value in our study population consisting of ELGA infants with relatively mild neuroimaging abnormalities. Only few SEP studies have paid attention to later cortical components. Using several recording electrodes, Karniski et al. $(23,24)$ noted that some SEP components with longer latencies were highly reproducible and suggested the 200 -ms component as a possible candidate in evaluating the status of somatosensory 


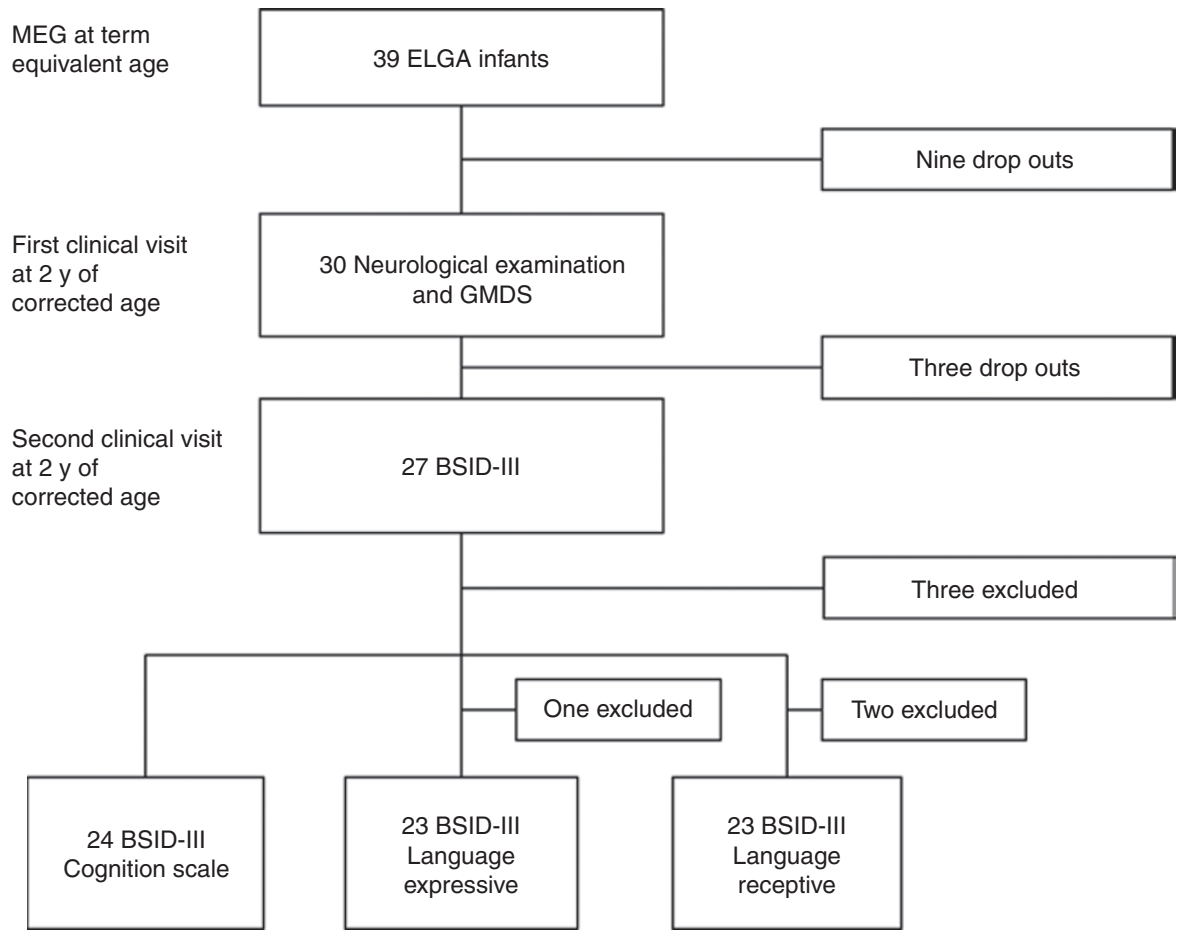

Figure 4. Study protocol, drop outs, and exclusions of extremely low-gestational-age (ELGA) infants. BSID-III, Bayley Scales of Infant and Toddler Development, Third Edition (31); GMDS, Griffiths Mental Development Scales (29); MEG, magnetoencephalography.

Table 5. Characteristics of study population

\begin{tabular}{lccc}
\hline & $\begin{array}{c}\text { ELGA infants } \\
(n=30)\end{array}$ & $\begin{array}{c}\text { Term controls } \\
(n=11)\end{array}$ & $P$ \\
\hline $\begin{array}{l}\text { Gestational age at } \\
\text { birth (wk) }\end{array}$ & $26.5(1.2)$ & $40.3(0.9)$ & $<0.001$ \\
Birth weight (g) & $884(181)$ & $3,605(482)$ & $<0.001$ \\
Male & $15[50.0]$ & $9[81.8]$ & 0.05 \\
SGA & $10[33.3]$ & 0 & 0.02 \\
Twins & $7[23.3]$ & 0 & 0.07 \\
Mother's age & $32.5[4.6]$ & $32.6[4.1]$ & 0.94 \\
Mother's educational & & & \\
attainment ${ }^{\mathrm{a}}$ & & & \\
$\quad$ Low & $1[3.8]$ & 0 & 0.55 \\
$\quad$ Middle & $15[57.7]$ & $4[44.4]$ & 0.49 \\
$\quad$ High & $10[38.5]$ & $5[55.6]$ & 0.37 \\
Cesarean section & $16[63.3]$ & $2[16.7]$ & 0.03 \\
\hline
\end{tabular}

Data shown as mean (SD) or $n[\%]$.

ELGA, extremely low gestational age; SGA, small for gestational age.

aData for four ELGA infants and two healthy term infants missing.

system in preterm infants $(23,24)$. In addition, in a preliminary MEG study in preterm infants, SII response was often absent at term in infants with anatomical lesion in the underlying hemisphere. However, in that study, the neurodevelopmental outcome of the infants was not available (18).

In the current study, the abnormal SII response in MEG was associated with poor total DQ and locomotor SQ in GMDS. Notably, among infants with severe IVH, two patients with normal SII response in MEG had markedly better neurodevelopmental outcome than one patient with severe IVH and abnormal SII response in MEG. Correspondingly, one infant with WM abnormalities in MRI and abnormal SII response in MEG had worse neurodevelopmental outcome than five infants with abnormal WM but normal SII response. These results highlight the complementary value of functional (neurophysiological) assessments in predicting neuromotor development after very preterm birth.

Although SI response in MEG or electroencephalography represents the intactness of pathways from the periphery to the SI, the SII response can be seen as an indicator of higher intracortical processing. Furthermore, the absence of SII response may reflect not only damage to the sensorimotor networks but possibly overall reduced corticocortical connectivity, which could be reflected in the total DQ. Behaviorally, SII neurons with bilateral receptive fields (25) have been suggested to integrate somatosensory information from the two body halves (26) and to integrate somatosensory and motor information (27), which makes the study of SII responses interesting, as ELGA infants have a high prevalence of minor neuromotor dysfunction and poor coordination (4).

In our study group, mild WM abnormalities in MRI were found in $26 \%$ of the ELGA infants, and there were no moderate-to-severe WM lesions, which is less than previously reported (mild: $58 \%$, moderate: $15 \%$, severe: $3 \%$ in infants born $<32$ gestational weeks) (28). The lower number of WM lesions in our study group is probably due to us being able to perform MEG recordings only on infants who did not require respiratory assistance or constant monitoring at TEA. Second, parents whose infants have had more complications during neonatal intensive care might be less willing to accept extra 
examinations of their infants than parents of infants without any noticeable complications. Consequently, more data are needed to generalize our SEF findings to ELGA infants with moderate-to-severe WM injury. Recent technical advances, such as infant-sized MEG devices and the development of analysis software to remove external magnetic artifacts and compensate for movements, should make this method more applicable in infants and perhaps even in neonatal intensive care unit settings.

A strength of our study is the homogeneous study population: all were born at $<28$ gestational weeks. The study population represents ELGA infants who received modern neonatal intensive care. Only $10 \%$ of infants had severe IVH, none had cystic periventricular leukomalacia, and $21 \%$ had mild WM abnormalities in MRI at TEA. Therefore, many of these ELGA infants left the neonatal intensive care unit without any visible morphological defects in US or MRI, but some of them still had an adverse neurodevelopmental outcome. Additional measures to recognize these infants as early as possible are needed.

A limitation of the study is the use of GMDS for children up to $2 \mathrm{y}$ of age, which created a ceiling effect on our GMDS results. This had no impact on results in infants with developmental impairments, but normally developed infants could have had better results if we had used the scales for infants older than $2 \mathrm{y}$. Therefore, the difference between infants with abnormal or normal SII response in DQ and subquotients in GMDS could have been larger without this ceiling effect. Furthermore, we could not measure all 39 ELGA infants using GMDS and BSID-III. In the study group, 30\% of ELGA infants had an abnormal SII response, whereas among nine infants excluded because of missing GMDS, five (55.6\%) had an abnormal SII response. Measuring the outcome of these nine excluded infants would have been valuable. Furthermore, the lack of information of neurological status at term precludes the comparison of MEG and clinical examination as outcome predictors. It would also have been interesting to evaluate the SII responses at $2 \mathrm{y}$ of age; however, at that age, MEG recordings, which require the subject to stay still, are extremely difficult to perform. Therefore, a follow-up MEG measurement is planned closer to school age.

This study demonstrated that the absence of SII responses to tactile stimulation, as measured by MEG, is associated with adverse neuromotor development at $2 \mathrm{y}$ of corrected age. The results suggest a role for the evaluation of the SII responses in the future as a part of developmental risk assessment for infants born extremely preterm.

\section{METHODS}

\section{Patients}

The subjects were 39 ELGA infants (born before 28 gestational weeks) recruited from the Neonatal Intensive Care Unit of the Helsinki University Central Hospital between May 2006 and September 2008 for a larger multimethodological study (84 ELGA infants and 22 term controls). The Ethics Committee of the Hospital District of Helsinki and Uusimaa approved the study protocol. The parents signed an informed consent. Exclusion criteria were the need for respiratory support or constant monitoring at TEA. Thirty-nine ELGA infants were eligible to undergo a MEG measurement at $37+6$ to $44+4$ wk of gestational age (mean: $41.1 \mathrm{wk})$. Of these infants, 30 also underwent GMDS (29) and a structured neurological examination (30) at $2 \mathrm{y}$ of corrected age. We excluded two uncooperative ELGA infants from BSID-III (31) Cognition Scale and one because the examination was not possible in her native language. In addition, two inattentive children failed to complete the Receptive Language Scale and one child the Expressive Language Scale. Figure 4 illustrates the drop outs and exclusions.

\section{Controls}

The controls were 11 healthy term infants recruited from the maternity ward of the Department of Obstetrics, Helsinki University Central Hospital. The controls underwent MEG recording 1-23 d after birth at the gestational age of $38+0$ to $43+1$ (mean: $41.0 \mathrm{wk}$ ). All controls were assessed at the age of $2 \mathrm{y}$ with GMDS and structured neurological examination, and nine of them also with BSIDIII. Table 5 presents the characteristics of the study population. There were more male infants in the control group than in the study group (Table 5).

\section{Clinical Data}

Obstetric and postnatal data of neonatal clinical course and complications were collected from the hospital records. Gestational age was based on the first-trimester US when available. Birth weight $z$-scores for gestational age and sex were based on the Finnish growth reference data (32). The highest grade of IVH in serial cranial US was recorded. Socioeconomic status was evaluated with a questionnaire given to the child's parents when the infant was $2 \mathrm{y}$ of age.

\section{Magnetoencephalography}

Stimulus. The tactile stimulus was given to the tip of the index finger by a thin elastic membrane expanded by an air pressure pulse delivered through a plastic tube (Somatosensory Stimulus Generator, 4-D NeuroImaging, San Diego, CA). For more details of the stimulation method, see Pihko et al. (33). The interstimulus interval was $2 \mathrm{~s}$.

Data acquisition. MEG was recorded in a magnetically shielded room (ETS-Lindgren Euroshield Oy, Eura, Finland) with a whole-head helmet-shaped sensor array (Vector View,Elekta Neuromag Oy, Helsinki, Finland) consisting of 306 independent channels: 204 gradiometers and 102 magnetometers. Electroencephalography with one to three electrodes and electro-oculography were simultaneously recorded for sleep-stage monitoring.

The helmet was in supine position and the infant lay with one hemisphere downward over the occipital part of the helmet. The stimulation and the recordings started when the infant fell asleep and stopped when the infant awoke; the infants were not sedated. In the beginning of each dataset, the infant's head position inside the sensor array was estimated using signals from position indicator coils attached to the infant's head. For more details of the recording settings and procedure, see Nevalainen et al. (18).

MEG data from the right hemisphere were recorded during stimulation of the contralateral left hand in all 30 ELGA infants included in the final study group and in 10 of 11 controls. Recordings from the left hemisphere during stimulation of the right index finger were obtained in 24 of 30 ELGA infants and 9 of 11 controls. Recordings during stimulation of the ipsilateral hand were obtained from the right hemisphere of 21 ELGA infants and from the left hemisphere of 20 of the 24 ELGA infants with data during contralateral stimulation. All conditions could not be obtained in all infants owing to restlessness of the infant and/or measurement time limitations.

Data analysis. A single MEG researcher (P.N.), who was blinded to the infants' neurodevelopmental outcome, analyzed all MEG data. The MEG data were first preprocessed with a spatiotemporal signal space separation method (MaxFilter,Elekta Neuromag Oy, Helsinki, Finland) to remove possible magnetic artifacts ( $8 \mathrm{~s}$ time window and 0.9 correlation limit) $(34,35)$. After spatiotemporal signal space separation, periods 
with movement artifacts were manually discarded from the data, and quiet sleep periods were averaged for further analyses. For details of the sleep-stage determination, see Pihko et al. (33). The averaged signals were baseline corrected ( -100 to $0 \mathrm{~ms}$ ) and low pass filtered at $90 \mathrm{~Hz}$.

From the quiet sleep data, the contralateral SI (M60) and contraand ipsilateral SII (M200) sources (17) were modeled with equivalent current dipoles using a spherical conductor model, which was individually constructed based on each infant's anatomical MRIs. When MRI was not available (one ELGA infant, one term infant), the average sphere origin, calculated from the other infants' MRIs, was used. When there was no clear peak or dipolar field pattern and/or the dipole could not be modeled with the required goodness of fit of $>70 \%$, the response was considered to be absent. Moreover, appropriate location of dipoles on individual MRIs was required for acceptance of SI and SII dipoles. For a detailed description of the dipole fitting procedure, see Nevalainen et al. (20).

\section{Magnetic Resonance Imaging}

Brain MRI (1.5T) including T2-weighted axial and T1-weighted three-dimensional sagittal images was performed near TEA on 29 of 30 ELGA infants in the study group and on 10 of 11 term controls. The images were classified by two experienced neuroradiologists (L.V. and T.A.) according to Woodward et al. (5).

\section{Neurodevelopmental Assessments}

The neurodevelopmental outcome was measured at $2 \mathrm{y}$ of corrected age by GMDS (29), a structured neurological examination (30), and BSIDIII (31), which were administered and scored by certified examiners. Neurological status and GMDS were assessed by a child neurologist (A.L.), who was unaware of the MEG and MRI classification results. If there were unscorable items or judgment was unreliable, the infant was classified as nontestable on that scale. GMDS provide an overall DQ and the quotients of locomotor, personal and social, hearing and language, eye-hand coordination and performance subscales (SQ). On the basis of neurological examination, infants were divided into four categories: normal, simple minor neurological dysfunction (MND-1), complex minor neurological dysfunction (MND-2), and cerebral palsy (36). Three subscales of five in BSID-III were scored (cognition, receptive language, and expressive language). Fine and gross motor in BSIDIII were omitted because the locomotor function (gross motor) and eye-hand coordination (fine motor) were assessed in GMDS.

\section{Statistical Analysis}

For continuous variables, unpaired comparisons were done with nonparametric Mann-Whitney $U$ test when data did not follow normal distribution (e.g., GMDS quotients) and with $t$-test when data followed normal distribution (e.g., BSID-III results). Categorical variables were compared by $\chi^{2}$ test. A $P$ value $<0.05$ was considered statistically significant.

\section{ACKNOWLEDGMENTS}

We are deeply indebted to Marita Suni for her excellence in caring for the very prematurely born infants during the MEG measurements. Furthermore, we express our gratitude to all our subjects and their families for preparing the way for this study. Finally, we thank the personnel of the maternity ward of the Department of Obstetrics and Gynecology and the Neonatal Intensive Care Unit at the Helsinki University Central Hospital, newborns' ward at Helsinki Maternity Hospital, and children's ward at Jorvi Hospital for their cooperation.

\section{STATEMENT OF FINANCIAL SUPPORT}

The study was financially supported by the Jenny and Antti Wihuri Foundation, the Academy of Finland (Centre of Excellence Program), the Finnish Medical Foundation, the Helsinki University Central Hospital Research Grants, Foundation for Pediatric Research, the Päivikki and Sakari Sohlberg Foundation, the Emil Aaltonen Foundation, the Maud Kuistila Memorial Foundation, and the aivoAALTO project of the Aalto University. S.V. was supported by the European Community's Seventh Framework Program European Community FP7-PEOPLE-2009-IOF, grant agreement no. 254235.

Disclosure: The authors declared no conflict of interest.

\section{REFERENCES}

1. Whittingham K, Wee D, Boyd R. Systematic review of the efficacy of parenting interventions for children with cerebral palsy. Child Care Health Dev 2011;37:475-83.

2. Mikkola K, Ritari N, Tommiska V, et al. Neurodevelopmental outcome at 5 years of age of a national cohort of extremely low birth weight infants who were born in 1996-1997. Pediatrics 2005;116:1391-400.

3. Salt A, Redshaw M. Neurodevelopmental follow-up after preterm birth: follow up after two years. Early Hum Dev 2006;82:185-97.

4. Saigal S, Doyle LW. An overview of mortality and sequelae of preterm birth from infancy to adulthood. Lancet 2008;371:261-9.

5. Woodward LJ, Anderson PJ, Austin NC, Howard K, Inder TE. Neonatal MRI to predict neurodevelopmental outcomes in preterm infants. N Engl J Med 2006;355:685-94.

6. Volpe JJ. Neurology of the Newborn, 5th edn. Philadelphia, PA: Saunders, 2008:172-74.

7. Miller SP, Ferriero DM, Leonard C, et al. Early brain injury in premature newborns detected with magnetic resonance imaging is associated with adverse early neurodevelopmental outcome. J Pediatr 2005;147:609-16.

8. Dyet LE, Kennea N, Counsell SJ, et al. Natural history of brain lesions in extremely preterm infants studied with serial magnetic resonance imaging from birth and neurodevelopmental assessment. Pediatrics 2006;118:53648.

9. Brown NC, Inder TE, Bear MJ, Hunt RW, Anderson PJ, Doyle LW. Neurobehavior at term and white and gray matter abnormalities in very preterm infants. J Pediatr 2009;155:32-8, 38.e1.

10. Skiöld B, Vollmer B, Böhm B, et al. Neonatal magnetic resonance imaging and outcome at age 30 months in extremely preterm infants. J Pediatr 2012;160:559-566.e1.

11. Mirmiran M, Barnes PD, Keller K, et al. Neonatal brain magnetic resonance imaging before discharge is better than serial cranial ultrasound in predicting cerebral palsy in very low birth weight preterm infants. Pediatrics 2004; 114:992-8.

12. Klimach VJ, Cooke RW. Short-latency cortical somatosensory evoked responses of preterm infants with ultrasound abnormality of the brain. Dev Med Child Neurol 1988;30:215-21.

13. Willis J, Duncan MC, Bell R, Pappas F, Moniz M. Somatosensory evoked potentials predict neuromotor outcome after periventricular hemorrhage. Dev Med Child Neurol 1989;31:435-9.

14. de Vries LS, Eken P, Pierrat V, Daniels H, Casaer P. Prediction of neurodevelopmental outcome in the preterm infant: short latency cortical somatosensory evoked potentials compared with cranial ultrasound. Arch Dis Child 1992;67(10 Spec No):1177-81.

15. Pierrat V, Eken P, de Vries LS. The predictive value of cranial ultrasound and of somatosensory evoked potentials after nerve stimulation for adverse neurological outcome in preterm infants. Dev Med Child Neurol 1997;39:398-403.

16. Volpe JJ. Brain injury in premature infants: a complex amalgam of destructive and developmental disturbances. Lancet Neurol 2009;8:110-24.

17. Nevalainen P, Lauronen L, Sambeth A, Wikström H, Okada Y, Pihko E. Somatosensory evoked magnetic fields from the primary and secondary somatosensory cortices in healthy newborns. Neuroimage 2008;40:73845.

18. Nevalainen P, Pihko E, Metsäranta M, Andersson S, Autti T, Lauronen L. Does very premature birth affect the functioning of the somatosensory cortex?-a magnetoencephalography study. Int J Psychophysiol 2008;68:8593.

19. Pihko E, Lauronen L, Kivistö K, Nevalainen P. Increasing the efficiency of neonatal MEG measurements by alternating auditory and tactile stimulation. Clin Neurophysiol 2011;122:808-14.

20. Nevalainen P, Pihko E, Metsäranta M, et al. Evoked magnetic fields from primary and secondary somatosensory cortices: a reliable tool for assessment of cortical processing in the neonatal period. Clin Neurophysiol 2012;123:2377-83.

21. Pike AA, Marlow N. The role of cortical evoked responses in predicting neuromotor outcome in very preterm infants. Early Hum Dev 2000;57:123-35. 
22. Vanhatalo S, Lauronen L. Neonatal SEP - back to bedside with basic science. Semin Fetal Neonatal Med 2006;11:464-70.

23. Karniski W. The late somatosensory evoked potential in premature and term infants. I. Principal component topography. Electroencephalogr Clin Neurophysiol 1992;84:32-43.

24. Karniski W, Wyble L, Lease L, Blair RC. The late somatosensory evoked potential in premature and term infants. II. Topography and latency development. Electroencephalogr Clin Neurophysiol 1992;84:44-54.

25. Whitsel BL, Petrucelli LM, Werner G. Symmetry and connectivity in the map of the body surface in somatosensory area II of primates. J Neurophysiol 1969;32:170-83.

26. Simões C, Hari R. Relationship between responses to contra- and ipsilateral stimuli in the human second somatosensory cortex SII. Neuroimage 1999;10:408-16.

27. Huttunen J, Wikström H, Korvenoja A, Seppäläinen AM, Aronen H, Ilmoniemi RJ. Significance of the second somatosensory cortex in sensorimotor integration: enhancement of sensory responses during finger movements. Neuroreport 1996;7:1009-12.

28. Woodward LJ, Clark CA, Pritchard VE, Anderson PJ, Inder TE. Neonatal white matter abnormalities predict global executive function impairment in children born very preterm. Dev Neuropsychol 2011;36:22-41.
29. Brandt I, Sticker EJ. Griffiths Entwicklungsskalen zur Beurteilung der Entwicklung in den ersten beiden Lebensjahren. Beltz Test GmbH, Göttingen, 2001.

30. Hempel MS. The Neurological Examination for Toddler Age. Thesis, University of Groningen: Groningen, The Netherlands, 1993.

31. Bayley N. The Bayley Scales of Infant and Toddler Development, Third Edition. San Antonio, TX: The Psychological Corporation, 2005.

32. Pihkala J, Hakala T, Voutilainen P, Raivio K. [Characteristic of recent fetal growth curves in Finland]. Duodecim 1989;105:1540-6.

33. Pihko E, Lauronen L, Wikström H, et al. Somatosensory evoked potentials and magnetic fields elicited by tactile stimulation of the hand during active and quiet sleep in newborns. Clin Neurophysiol 2004;115:448-55.

34. Taulu S, Simola J. Spatiotemporal signal space separation method for rejecting nearby interference in MEG measurements. Phys Med Biol 2006;51:1759-68.

35. Medvedovsky M, Taulu S, Bikmullina R, Ahonen A, Paetau R. Fine tuning the correlation limit of spatio-temporal signal space separation for magnetoencephalography. J Neurosci Methods 2009;177:203-11.

36. Hadders-Algra M. Developmental coordination disorder: is clumsy motor behavior caused by a lesion of the brain at early age? Neural Plast 2003;10:39-50. 